\title{
Adjustable Gastric Banding for Morbid Obesity; A Report from Iran
}

\author{
Mojtaba Hashemzadeh ${ }^{1,2}$, Leila Zahedi-Shoolami ${ }^{2, *}$ and Mahmoud KaramiRad ${ }^{3}$

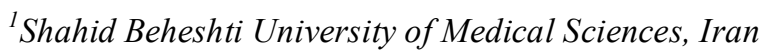 \\ ${ }^{2}$ Tehran University of Medical Sciences, Iran \\ ${ }^{3}$ Kermanshah University of Medical Sciences, Iran
}

\begin{abstract}
Background: Laparoscopic adjustable gastric banding (LAGB) was considered as one of the effective, less invasive methods which could help the obese patients to achieve their optimal weight without regaining the lost one. The recent article is a report of 165 patients who had undergone LAGB, while discussing the rate of their complications.

Methods: Between January 2005 and February 2012, 165 obese patients had undergone LAGB in a single private surgical setting in Tehran, Iran. Patients with body mass indices (BMI) between 32 and $50 \mathrm{~kg} / \mathrm{m}^{2}$ were candidates for the LAGB operation. The subsequent complications were recorded during the patient's follow-up periods.

Results: The patients' mean initial BMI was $37 \pm 0.3 \mathrm{~kg} / \mathrm{m}^{2}$ which became $29.4 \pm 0.3 \mathrm{~kg} / \mathrm{m}^{2}$ after LAGB. The mean weight reduction was $21.4 \pm 0.9 \mathrm{~kg}$ during the $16.9 \pm 0.9$ months follow-up period. Morbidity rate requiring reoperation was $9 \%$ (slippage, erosion and phrenic nerve irritation. Thirty-nine patients experienced complications, including 21 slippages, 11 port infections, six erosions and one phrenic irritation.

Conclusions: Despite the complications being reported, LAGB is still a safe method with an acceptable efficacy in losing weight. To our knowledge, the important factor to obtain the appropriate response is selecting cases precisely. The complications could be managed by surgeons who are experienced in upper-gastrointestinal laparoscopic surgery. Otherwise patients need to be operated through laparotomy or referred to the bariatric surgeons.
\end{abstract}

Keywords: Complications, Adjustable Gastric Banding, Morbid Obesity.

\section{INTRODUCTION}

In Iran the prevalence of obesity varies due to different ethnic groups of Turks, Kurds, Arabs, Fars, Turkmen and Baluchs and also a wide variety of cultures, life styles and socio-economic status [1].

According to the 2011 report of World Health Organization about non-communicable diseases in Iran, being overweight or obese were considered as two main metabolic risk factors among Iranians, with an estimated prevalence of $56.8 \%$ and $26.5 \%$, respectively with significant dominancy in women [2].

The results of meta-analysis on national surveys also demonstrated a $21.5 \%$ of obesity (BMI $\geq 30$ ) among adults ( $\geq 18$ years old).The increasingly prevalence of obesity, besides the lack of an integrated or topic-specific operational policy and action plan on unhealthy diet and overweight makes it crucial to consider practical approaches towards decreasing the risk in population [3].

Following the inconstant success of the non-surgical modalities in the treatment of obesity, including dietary changes and exercise, various surgical techniques have been introduced in order to reduce the excess weight and decrease

*Address correspondence to this author at the Iran-Tehran-Yousefabad Street, $47^{\text {th }}$ Avenue, Nader Building, No. 204; Tel: 0098-9123789100;

E-mail: Leila.Zahedi@gmail.com the comorbidity rates of the obesity. Laparoscopic adjustable gastric banding (LAGB) was considered as one of the effective, less invasive methods which could help the obese patients to achieve their optimal weight without regaining the lost one. But unfortunately besides the advantages and benefits of the LAGB, the presence of complications such as the band slippage, gastric erosion and port-infection have complicated the patient's situation in a way which prompt assessment and strict management modalities were necessary in order to save the patient's life.

The recent article is a report of 165 Iranian patients who had undergone LAGB, while discussing the rate of their complications.

\section{MATERIALS AND METHODS}

Between January 2005 and February 2012, obese patients had undergone LAGB in a single private surgical setting in Tehran, Iran. Patients with body mass indices (BMI) between 32 and $50 \mathrm{~kg} / \mathrm{m}^{2}$ were candidates for the LAGB operation. A file was considered for each patient and all the presurgical and follow-up evaluations and complications were recorded precisely.

\section{PREOPERATIVE MANAGEMENT}

During the first visit in the surgeon's office, patients underwent a preoperative evaluation including a thorough history, physical exam and a psychiatric, nutritional and diet status evaluation. If the patients were the candidates of the 
Table 1. Outcome of Comorbidity

\begin{tabular}{|c|c|c|c|c|c|}
\hline & Number of Comorbidities & Complete Improvement & Relative Improvement & No Change & Getting Worse \\
\hline \hline Dyslipidemia & 14 & $\mathrm{a} 4$ & 7 & 3 & 0 \\
\hline Diabetes & 7 & 1 & 6 & 0 & 2 \\
\hline Hypertension & 6 & 3 & 1 & 0 \\
\hline Sleep Apnea & 4 & 2 & 0 & 0 \\
\hline Fatty Liver & 3 & 3 & 0 & 0 \\
\hline Degenerative Joint Disease & 2 & 1 & 1 & 0 \\
\hline
\end{tabular}

LAGB, the operation procedure, advantages and disadvantages, the potential risks and the post-operation issues were discussed in details and all questions were answered precisely. Dietary restrictions and the need for frequent post-operation visits were also explained for the patients. A two weeks interval was mandatory before making any decision and patients were encouraged to complete their information about the procedure and the risks via previously operated patients. During this period, a thorough laboratory test, including complete blood count (CBC), serum biochemical analysis, liver function tests and lipid profile, thyroid function tests, Dexamethasone suppression test, Beta hCG, blood group and $\mathrm{Rh}$ and also an abdominal ultrasonography were ordered for all patients. Cardiologic and respiratory consult, besides an anesthesiologist visit, were also performed for all patients prior to surgery.

During the second visit and after evaluating the patient's laboratory tests, sonography and the consultation results, and in case of the patient's wish to undergo the LAGB, a written consent was obtained, in which the patient accepted the previously discussed risks and side effects of the procedure. Afterward the patients were planned for LAGB at the earliest available time schedule. The technique was performed through laparoscopy and general anesthesia.

\section{OPERATIVE TECHNIQUE}

The procedure was performed under general anesthesia and the patient was tilted in the reverse Trendelenburg position. The surgeon stood between the legs of the patient, while the cameraman was at his right and the assistant was at his left side.

The camera was inserted through the umbilical $10-\mathrm{mm}$ port and the liver retractor through the 5-mm xyphoid port. A 16-mm trocar was inserted below the costal margin in the left upper quadrant at the midclavicular line and another 5$\mathrm{mm}$ trocar was placed on the right midclavicular side, mirroring the previously mentioned one in the left side.

After introducing the trocars into the abdominal cavity, the abdomen was insufflated with a mean intraabdominal pressure of $17 \mathrm{mmHg}$.

In the next step the ligaments and attachments were released at the His angle while a window was created on the pars flaccida, at the right crus in order to access the retrogastric space to pass the band's tube. The band (Helioscope or Cousin Bioring) was introduced to the abdomen through the $16 \mathrm{~mm}$ port into the abdomen while the end of the tubing was passed behind the stomach, and the band was locked in placed upon itself. The fundus of the stomach was then fixed to the abdominal esophagus over the band with a single suture.

After fixing the gastric band, the anesthesiologist injected methylene blue dye through the nasogastric tube which was placed earlier, in order to detect any probable perforations in the gastric field of surgery. After removing the liver retractor and trocars, incisions were closed in the classic form.

The band's connecting tube which was extracted from the 16-mm port site was then shortened and fixed to the band's injection port. For fixing the port a new set of sterile gloves were used and the port was fixed to the rectus fascia in the midline with a $5-\mathrm{mm}$ distance from the $16-\mathrm{mm}$ trocar site. Two injections of Gentamycine antibiotic were performed directly beside the port site.

Patients were assessed 6 hours later and in case of toleration, fluids were started.

In the absence of complications, patients were discharged from the hospital in less than 24 hours after the surgery. Three days and one week later patients were visited in the office. Three weeks later (4 weeks post-op), the first injection was performed in order to adjust the gastric banding. To our experience injecting saline was practically difficult in Helioscope ports and the bands were inflated with sterile water. The subsequent injections were performed considering the patient's clinical evaluation and weight loss during the follow-ups.

\section{FOLLOW-UPS}

Follow-ups were performed upon patient's request. The adjustments were performed in office. The decision to do an adjustment was related to how hungry the patient was feeling, how much weight he or she was losing, and what he or she was eating. There was no specified number of adjustments. The dietary behavior of the Iranians and the taste for good meals made us to educate patients about low calorie diets and restrictions of sweets and alcohol. In each follow-up visit patients were reminded of the eating habits and were recommended to have small bites of food while avoiding pepper and additives.

\section{RESULTS}

There were $24(14.5 \%)$ men and $141(85.5 \%)$ women with a mean BMI of $37 \pm 0.3 \mathrm{~kg} / \mathrm{m}^{2}$ and the mean age of $32.8 \pm 0.7$ years. A total of 36 comorbidities were reported in 165 patients. The most common reported comorbidities were hyperlipidemia, diabetes mellitus, hypertension, sleep apnea, fatty liver and degenerative joint disease. The detailed list of comorbidities is demonstrated in Table $\mathbf{1}$. 
Table 2. Comparison of the Weight Reduction Indices Between the Patients with $\mathrm{BMI}<40 \mathrm{~kg} / \mathrm{m}^{2}$ and $\mathrm{BMI} \geq 40 \mathrm{~kg} / \mathrm{m}^{2}$

\begin{tabular}{|l|c|c|c|}
\hline & BMI $<\mathbf{4 0 ~} \mathbf{~ k g} / \mathbf{m}^{\mathbf{2}}$ & BMI $\geq \mathbf{4 0 ~} \mathbf{~ k g} / \mathbf{m}^{\mathbf{2}}$ & P Value \\
\hline \hline Weight Reduction & $19.1 \pm 0.9$ & $27.6 \pm 2$ & $0.00^{*}$ \\
\hline Pre-operation BMI & $34.8 \pm 0.2$ & $42.8 \pm 0.4$ & $0.00^{*}$ \\
\hline Post-operation BMI & $27.9 \pm 0.3$ & $33.2 \pm 0.8$ & $0.00^{*}$ \\
\hline BMI Reduction & $6.8 \pm 0.3$ & $9.6 \pm 0.6$ & $0.00^{*}$ \\
\hline Weight Loss \% & $19.4 \pm 0.8$ & $22.6 \pm 1.4$ & 0.06 \\
\hline Excess Weight Loss \% & $28.6 \pm 1.3$ & $40.3 \pm 2.5$ & $0.00^{*}$ \\
\hline
\end{tabular}

$* p<0.05$ is considered significant.

Table 3. Comparison of the Complications Between the Patients with BMI $<40 \mathrm{~kg} / \mathrm{m}^{2}$ and BMI $\geq 40 \mathrm{~kg} / \mathrm{m}^{2}$

\begin{tabular}{|l|c|c|c|}
\hline & BMI $<\mathbf{4 0} \mathbf{~ k g} / \mathbf{m}^{2}$ & BMI $\geq \mathbf{4 0} \mathbf{~ k g} / \mathbf{m}^{\mathbf{2}}$ & P Value \\
\hline \hline No Complications & $84(72.4)$ & $35(77.8)$ & \\
\cline { 1 - 3 } Slippage & $18(15.5)$ & $3(6.7)$ & \\
\cline { 1 - 3 } Port-site infection & $8(6.9)$ & $3(6.7)$ & \multirow{3}{*}{0.7} \\
\hline Erosion & $3(2.6)$ & $3(6.7)$ & \\
\hline Phrenic Nerve Irritation & 1 & 0 & \\
\hline
\end{tabular}

The mean operation time from the insertion of the first port to the final suture was $20.1 \pm 2.3$ minutes. Conversion to laparotomy was necessary in one of the cases due to right hepatic artery trauma and three blood unit transfusions were needed. No deaths had occurred during or post operation.

Concomitant cholecystectomy was performed in seven cases. The xyphoid trocar had been replaced with a $10-\mathrm{mm}$ one and the gall bladder was extracted through the xyphoid port. No infection in the port place was found.

The mean hospital stay was $17.8 \pm 0.8$ hours and the mean time to return to work was $6.1 \pm 0.4$ days.

The patients' mean initial BMI was $37 \pm 0.3 \mathrm{~kg} / \mathrm{m}^{2}$ which became $29.4 \pm 0.3 \mathrm{~kg} / \mathrm{m}^{2}$ after LAGB.

The median of percentage of weight loss ( $\% \mathrm{WL})$ and excess body weight loss (EBWL) was 20.5 and $32 \%$, respectively. EBWL was calculated based on Ideal Weight. The mean weight reduction was $21.4 \pm 0.9 \mathrm{~kg}$.

Thirty-nine patients experienced complications, including $21(12.7 \%)$ slippages, $11(6.6 \%)$ port infections,6 $(3.6 \%)$ erosions and one $(0.6 \%)$ phrenic nerve irritation during the $16.9 \pm 0.9$ months follow-up period. Morbidity rate requiring reoperation was $9 \%$ (slippage, erosion and phrenic nerve irritation).

Patients with $\mathrm{BMI} \geq 40 \mathrm{~kg} / \mathrm{m}^{2}$ were compared to those with BMI less than $40 \mathrm{~kg} / \mathrm{m}^{2}$. Significant difference was noted in the weight loss between these two groups. (Table 2) The incidence of complications was not significantly different between these groups (Table 3 ).

While asking the patients if they would recommend the technique to the other patients or not $27(16.9 \%)$ answered "No", 126 (78.8\%) answered "Yes Definitely" and 7 (4.4\%) answered "Yes Probably".

\section{DISCUSSION}

We represent a total number of 165 patients, with a mean follow-up period of $16.9 \pm 0.9$ months who had undergone LAGB. Our results indicated that LAGB is an effective method in managing the obese patients. Similar to the previous studies no mortality or death was observed in our population group. [4-6]

Despite fixing the band to the esophageal junction [7], slippage had occurred in $21(12.7 \%)$ cases. In all cases with slippage, frequent vomiting both caused by swallowing large portions of food or self-induced vomiting was observed. The literature reviews revealed a $1-22 \%$ of band slippages post LAGB operation [8-14]. Thirteen patients were managed conservatively by complete emptying of the gastric band while prescribing Proton Pump Inhibitors simultaneously for about 12 weeks. The remained eight patients in whom conservative management was failed, readjustment of the gastric band were performed through laparoscopy.

Among the six patients who had experienced erosion, three had mild gastric mucosal inflammation without significant band erosion into the stomach cavity. The bands were deflated and oral antibiotics and proton pump inhibitors were administered for two weeks. The second look through endoscopy, two weeks later, revealed complete healing of the inflammation.

The three other patients with gross band erosion in the endoscopy had undergone laparoscopic reoperation in which the gastric band was removed. Considering the reported prevalence of band erosion which varies from $0-11 \%$ in literature, our $3.6 \%$ incidence of this complication was comparatively low $[5,8]$.

Late port-site infection manifested by port swelling and pain was another major complication occurred in $6.6 \%$ of our patients. Patients with late port-site infection were all undergone endoscopy. In the absence of gastric band erosion (eight among the total of 11), patients received a ten day period of antibiotics besides completely deflating the gastric band. After the ten-day period, the port was removed through a small local incision and the gastric tube was fixed to the fascia. The new sterile port was replaced three months later, after the definite eradication of infection. The remained three patients with late port-site infection had erosions in 
their endoscopy and the bands were removed through laparoscopy.

Insufficient weight loss was mainly due to the failure in changing the eating habits, using high-calorie diets including sweets and alcohol, failure to change the sedentary life style and lack of exercise. Most of the patients confessed about the accuracy of the above mentioned reasons.

Despite the complications being reported, LAGB is still a safe method with an acceptable efficacy in losing weight. To our knowledge, the important factor to obtain the appropriate response is selecting cases precisely. The complications could be managed by surgeons who are experienced in upper-gastrointestinal laparoscopic surgery. Other-wise patients need to be operated through laparotomy or referred to the bariatric surgeons.

\section{CONFLICT OF INTEREST}

The authors confirm that this article content has no conflicts of interest.

\section{ACKNOWLEDGEMENT}

Declared none.

\section{REFERENCES}

[1] Iran Statistical Year Book. Tehran, Iran: The Statistical Center of Iran, Tehran, 2006.

[2] Noncommunicable diseases country profiles 2011, WHO global report 2011. Available from: http://whqlibdoc.who.int/publications/2011/9789241502283_eng.pdf.
[3] Mirzazadeh A, Sadeghirad B, Haghdoost A, Bahreini F, Kermani MR. The prevalence of obesity in Iran in recent decade; a systematic review and meta-analysis study. Iranian J Public Health 2009; 38: 1-11.

[4] Baraket O, El Ajmi M, Chouchene A, et al. Results of laparoscopic treatment of morbid obesity: report of 27 cases. Tunis Med 2010; 88: 799-803.

[5] Eid I, Birch DW, Sharma AM, Sherman V, Karmali S. Complications associated with adjustable gastric banding for morbid obesity: a surgeon's guides. Can J Surg 2011; 54: 61-6.

[6] Vijgen GH, Schouten R, Pelzers L, et al. Revision of laparoscopic adjustable gastric banding: success or failure? Obes Surg 2012; 22: 287-92.

[7] Avsar FM, Sakcak I, Yildiz BD, Cosgun E, Hamamci EO. Is gastro-gastric fixation suture necessary in laparoscopic adjustable gastric banding? A prospective randomized study. J Laparoendosc Adv Surg Tech A 2011; 21: 953-6.

[8] Abu-Abeid S, Szold A. Laparoscopic management of Lap-Band erosion. Obes Surg 2001; 11: 87-9.

[9] Alhamdani A, Wilson M, Jones T, et al. Laparoscopic adjustable gastric banding: a 10-year single-centre experience of 575 cases with weight loss following surgery. Obes Surg 2012; 22: 1029-38.

[10] Fielding GA, Ren CJ. Laparoscopic adjustable gastric band. Surg Clin North Am 2005; 85: 129-40.

[11] Martikainen T, Pirinen E, Alhava E, et al. Long-term results, late complications and quality of life in a series of adjustable gastric banding. Obes Surg 2004; 14: 648-54.

[12] O'Brien PE, Dixon JB. Weight loss and early and late complications--the international experience. Am J Surg 2002; 184 42-5.

[13] Sarker S, Herold K, Creech S, Shayani V. Early and late complications following laparoscopic adjustable gastric banding. Am Surg 2004; 70: 146-50.

[14] Tran D, Rhoden DH, Cacchione RN, Baldwin L, Allen JW Techniques for repair of gastric prolapse after laparoscopic gastric banding. J Laparoendosc Adv Surg Tech A 2004; 14: 117-20.

(C) Hashemzadeh et al.; Licensee Bentham Open.

This is an open access article licensed under the terms of the Creative Commons Attribution Non-Commercial License (http://creativecommons.org/licenses/by-nc/3.0/) which permits unrestricted, non-commercial use, distribution and reproduction in any medium, provided the work is properly cited. 